\title{
The Geoengineering Model Intercomparison Project Phase 6 (GeoMIP6): simulation design and preliminary results
}

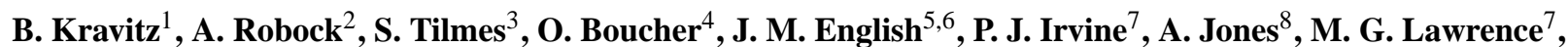
M. MacCracken ${ }^{9}$, H. Muri ${ }^{10}$, J. C. Moore ${ }^{11}$, U. Niemeier ${ }^{12}$, S. J. Phipps ${ }^{13}$, J. Sillmann ${ }^{14}$, T. Storelvmo ${ }^{15}$, H. Wang ${ }^{1}$, and S. Watanabe ${ }^{16}$

${ }^{1}$ Atmospheric Sciences and Global Change Division, Pacific Northwest National Laboratory, Richland, WA, USA

${ }^{2}$ Department of Environmental Sciences, Rutgers University, New Brunswick, NJ, USA

${ }^{3}$ National Center for Atmospheric Research, Boulder, CO, USA

${ }^{4}$ Laboratoire de Météorologie Dynamique, IPSL, CNRS/UPMC, Paris, France

${ }^{5}$ NOAA Earth System Research Laboratory, Boulder, CO, USA

${ }^{6}$ Cooperative Institute for Research in Environmental Sciences, University of Colorado, Boulder, CO, USA

${ }^{7}$ Institute for Advanced Sustainability Studies, Potsdam, Germany

${ }^{8}$ Met Office Hadley Centre, Exeter, UK

${ }^{9}$ Climate Institute, Washington, D.C., USA

${ }^{10}$ Department of Geosciences, University of Oslo, Oslo, Norway

${ }^{11}$ Joint Center for Global Change Studies, College of Global Change and Earth System Science, Beijing Normal University, Beijing, China

${ }^{12}$ Max Planck Institute for Meteorology, Hamburg, Germany

${ }^{13}$ ARC Centre of Excellence for Climate System Science and Climate Change Research Centre,

University of New South Wales, Sydney, Australia

${ }^{14}$ Center for International Climate and Environmental Research, Oslo, Norway

${ }^{15}$ Department of Geology and Geophysics, Yale University, New Haven, CT, USA

${ }^{16} \mathrm{Japan}$ Agency for Marine-Earth Science and Technology, Yokohama, Japan

Correspondence to: B. Kravitz (ben.kravitz@pnnl.gov)

Received: 7 May 2015 - Published in Geosci. Model Dev. Discuss.: 22 June 2015

Revised: 1 October 2015 - Accepted: 14 October 2015 - Published: 27 October 2015

\begin{abstract}
We present a suite of new climate model experiment designs for the Geoengineering Model Intercomparison Project (GeoMIP). This set of experiments, named GeoMIP6 (to be consistent with the Coupled Model Intercomparison Project Phase 6), builds on the previous GeoMIP project simulations, and has been expanded to address several further important topics, including key uncertainties in extreme events, the use of geoengineering as part of a portfolio of responses to climate change, and the relatively new idea of cirrus cloud thinning to allow more longwave radiation to escape to space. We discuss experiment designs, as well as the rationale for those designs, showing preliminary results from individual models when available. We also introduce a new feature, called the GeoMIP Testbed, which
\end{abstract}

provides a platform for simulations that will be performed with a few models and subsequently assessed to determine whether the proposed experiment designs will be adopted as core (Tier 1) GeoMIP experiments. This is meant to encourage various stakeholders to propose new targeted experiments that address their key open science questions, with the goal of making GeoMIP more relevant to a broader set of communities. 


\section{Introduction}

As anthropogenic climate change continues largely unabated, society is exploring research into options for addressing the effects of greenhouse gas emissions. Along with mitigation and adaptation, a further option that is under consideration is geoengineering, a term describing deliberate modification of the climate system to offset the radiative effects of increasing anthropogenic greenhouse gases. Geoengineering, in its usual definition, also includes proposals for greenhouse gas removal, but in this paper we will use the term "geoengineering", in the context of the Geoengineering Model Intercomparison Project (GeoMIP), to specifically refer to a broad range of proposed techniques that do not directly attempt to increase the carbon sink. Better understanding the potential role that geoengineering might have in addressing climate change requires research on the climate effects and impacts, as well as the underlying processes involved and their uncertainties.

The goal of GeoMIP is to understand the robust climate model responses to geoengineering (Kravitz et al., 2011). So far, there have been seven core climate model experiments designed for analyzing the effects of solar irradiance reduction, an increase in the loading of stratospheric sulfate aerosols, and marine cloud (or sky) brightening (Kravitz et al., 2011, 2013a), as well as several additional experiments proposed by various groups. Table 1 lists all of the proposed experiments to date. GeoMIP has achieved success on a number of fronts: 15 modeling groups have participated in one or more experiments. As of the writing of this paper, GeoMIP has resulted in 23 peer-reviewed publications; and results from GeoMIP were featured in the Fifth Assessment Report of the Intergovernmental Panel on Climate Change (Boucher et al., 2013), the recent National Academy of Sciences report on SRM (solar radiation management; NAS, 2015), and the final report from the European Transdisciplinary Assessment of Climate Engineering (EuTRACE).

These past efforts targeted specific areas. However, they were not designed to answer all questions about the potential climate effects of geoengineering, including questions about geoengineering methods that have been proposed, and remaining unanswered questions about conduct and design of research activities. The Coupled Model Intercomparison Project is beginning its sixth phase (CMIP6), and one of its focus areas is geoengineering (Meehl et al., 2014). Now is an opportune moment to address some of the key uncertainties regarding geoengineering by introducing designs for a new suite of climate modeling experiments. Pressing questions we propose to address include the following:

1. How would geoengineering affect changes in less easily detectable climate features, such as extreme events, modes of natural variability, regional impacts, and long timescale processes?
2. Cirrus cloud thinning is a newly proposed geoengineering method. What are the common responses in its simulation?

3. How would the climate response to geoengineering differ if it were used to slow rather than halt climate change? That is, what are common responses in climate models if geoengineering were to be used to only partially offset climate change?

4. What are robust differences in the climate model response between stratospheric sulfate aerosol injection and solar irradiance reduction?

In this paper, we outline four Tier 1 experiments for the next phase of GeoMIP, which, to be consistent with the numbering convention of CMIP, we call GeoMIP6. The experiment design for GeoMIP6 is based on discussions held at the Fourth GeoMIP Workshop (Paris, April 2014; Kravitz et al., 2014a), the SCRiM (Sustainable Climate Risk Management) All Hands Meeting (State College, May 2014), and the Exploring the Potential and Side Effects of Climate Engineering (EXPECT) workshop (Oslo, June 2014), as well as an experiment proposed for inclusion in the Chemistry Climate Model Initiative (CCMI; Tilmes et al., 2015). All of the proposed experiments are listed in Table 1 along with all previous GeoMIP and GeoMIP-affiliated experiments.

The guiding science questions in GeoMIP6 are directly relevant to the core questions of CMIP6. Geoengineering simulations have repeatedly been shown to be a novel method of uncovering fundamental climate behavior (e.g., Kleidon et al., 2015; Kravitz et al., 2013b) and continue to be relevant for addressing the question, "How does the Earth System respond to forcing?" Experiment G1 has already proven quite useful in this regard, particularly in its ability to separate mechanistic changes that contribute to the fast and slow responses of the climate system (e.g., Kravitz et al., 2013b; Tilmes et al., 2013); G1ext will likely provide even more information about mechanistic changes in the climate system slow response. Experiments G6sulfur and G6solar (below) will provide a useful multi-model comparison of the Earth system response to different forcing agents in a controlled protocol. GeoMIP has also been successful in identifying both model commonalities and the effects of different stratospheric aerosol parameterizations on the climate effects of geoengineering (e.g., Berdahl et al., 2014; Yu et al., 2015). These efforts are continuing for sea spray geoengineering experiments (Kravitz et al., 2013a). Our experimental design, particularly for G6sulfur and G7cirrus (below), will aid in uncovering the origins and consequences of different model parameterizations and how they contribute to model biases. Geoengineering simulations have been shown to actually reduce certain aspects of climate uncertainty and sources of model bias (Kravitz et al., 2013c; MacMartin et al., 2015). As such, we see our efforts as highly synergistic with those of CMIP6, potentially providing relevant information 
Table 1. All core GeoMIP experiments up to this point, including the additional proposed Tier 1 GeoMIP6 experiments. Only the time-slice Tier 2 experiments are listed in Table 2. For each experiment, the name is given, along with a short description and reference. Newly proposed experiments are printed in boldface. G5 is not a core GeoMIP experiment but is included for completeness.

\begin{tabular}{|c|c|c|}
\hline Experiment name & Description & Reference \\
\hline G1 & Balance $4 \times \mathrm{CO}_{2}$ via solar irradiance reduction & Kravitz et al. (2011) \\
\hline G1ext & Same as G1 but extended an extra 50 years & This document \\
\hline G1ocean-albedo & Balance $4 \times \mathrm{CO}_{2}$ via ocean albedo increase & Kravitz et al. (2013a) \\
\hline G2 & Balance $1 \% \mathrm{CO}_{2}$ increase per year via solar irradiance reduction & Kravitz et al. (2011) \\
\hline G3 & $\begin{array}{l}\text { Keep TOA radiative flux at } 2020 \text { levels against RCP4.5 via strato- } \\
\text { spheric sulfate aerosols }\end{array}$ & Kravitz et al. (2011) \\
\hline G4 & Injection of $5 \mathrm{Tg} \mathrm{SO}_{2}$ into lower stratosphere per year & Kravitz et al. (2011) \\
\hline G4cdnc & $\begin{array}{l}\text { Increase CDNC (cloud droplet number concentration) in marine } \\
\text { low clouds by } 50 \% \text { against a background of RCP4.5 }\end{array}$ & Kravitz et al. (2013a) \\
\hline G4sea-salt & $\begin{array}{l}\text { Inject sea salt aerosols into tropical marine boundary layer to } \\
\text { achieve ERF* of }-2.0 \mathrm{~W} \mathrm{~m}^{-2} \text { against a background of RCP4.5 }\end{array}$ & Kravitz et al. (2013a) \\
\hline G5 & $\begin{array}{l}\text { Identical setup as G3 but using sea salt injection into marine low } \\
\text { clouds (IMPLICC experiment; named SALT in Niemeier et al., } \\
\text { 2013) }\end{array}$ & $\begin{array}{l}\text { Alterskjær et al. (2013); } \\
\text { Niemeier et al. (2013) }\end{array}$ \\
\hline G6sulfur & $\begin{array}{l}\text { Reduce forcing from ScenarioMIP Tier } 1 \text { high forcing sce- } \\
\text { nario to the medium forcing scenario with stratospheric sulfate } \\
\text { aerosols }\end{array}$ & This document \\
\hline G6solar & $\begin{array}{l}\text { Reduce forcing from ScenarioMIP Tier } 1 \text { high forcing scenario } \\
\text { to the medium forcing scenario with solar irradiance reduction }\end{array}$ & This document \\
\hline G7cirrus & $\begin{array}{l}\text { Reduce forcing by constant amount (against a baseline of the } \\
\text { ScenarioMIP Tier } 1 \text { high forcing scenario) via increasing cirrus } \\
\text { ice crystal fall speed }\end{array}$ & This document \\
\hline
\end{tabular}

* ERF - effective radiative forcing.

to the driving science questions via relatively underexplored means.

\section{Tier 1 experiments in GeoMIP6}

In this section, we outline the four Tier 1 experiments that are proposed for GeoMIP6. These same experiments have also been proposed for inclusion in CMIP6, with GeoMIP serving as an officially endorsed model intercomparison project.

The general experimental protocol is somewhat different from that of the previous experiments (Kravitz et al., 2011, 2013a; also see Table 1). There has recently been interest in conducting geoengineering studies that examine phenomena for which previous experiments have generated only a low signal-to-noise ratio, for example, extreme temperature and precipitation events (Curry et al., 2014) and modes of internal variability (Gabriel and Robock, 2015). To obtain more robust estimates of potential changes in extreme events and regional climate, we are now requesting that all simulations be conducted for longer than 50 years. Cessation or termination (in which the background scenario continues, but geoengineering is no longer conducted) is no longer part of the experimental protocol. Many of the broad messages associ- ated with the so-called termination effect were well captured by Jones et al. (2013), so additional efforts to represent termination are not currently a high priority.

The monthly average output requested for each experiment should be the same as is requested for the core CMIP6 experiments (see below). In addition, we request that all modeling groups produce the following at daily frequency: minimum and maximum near-surface air temperature (reference height; usually $1.5-2 \mathrm{~m}$ ), total surface precipitation, surface convective precipitation, near-surface (usually $10 \mathrm{~m}$ ) wind speed, and hourly surface ozone concentration, if available. If possible, precipitation and convective precipitation should be reported as a cumulative value at 6-hourly frequency, and wind speed should be reported as an instantaneous value at 6-hourly frequency. Each modeling group should produce a minimum of three ensemble members for each experiment; ideally, groups would complete five or more ensemble members.

As before, the Tier 1 experiments will be based on core experiments in CMIP. The newest version of the core CMIP6 experiments is called the CMIP Diagnostic, Evaluation and Characterization of Klima (DECK) experiment portfolio (Meehl et al., 2014). This will include many different sim- 
ulations, but the DECK simulations that are relevant for GeoMIP6 are piControl, historical, and abrupt $4 \mathrm{xCO} 2$, each of which was also included in CMIP5. Additionally, simulations involving future projections of climate change scenarios will be based on the Tier 1 simulations of ScenarioMIP (O'Neill et al., 2014). Tier 1 of ScenarioMIP will consist of high, medium, and low forcing scenarios, referring to the magnitude of anthropogenic radiative forcing applied in that scenario.

\subsection{G1ext}

This experiment is planned as an extended version of experiment G1 (Kravitz et al., 2011). G1ext proposes that, beginning from a preindustrial simulation (piControl), the net top-of-atmosphere (TOA) radiative flux imbalance due to an abrupt quadrupling of the $\mathrm{CO}_{2}$ concentration (abrupt $4 \mathrm{xCO} 2$ ) would be balanced via a reduction in total solar irradiance (Fig. 1). Here, "balance" is defined as the global mean value top-of-atmosphere net radiative flux being within $\pm 0.1 \mathrm{~W} \mathrm{~m}^{-2}$ of the piControl experiment over an average of years $1-10$ of the simulation. The original G1 was conducted for 50 simulation years, so this will be a simple extension of the previous experiment. Modeling groups that have already moved on to a new model version, or for whatever reason are not able to extend their previous model run, should run experiment G1ext for the full 100 years with their new version.

G1 has proven quite successful in revealing the underlying climate behavior in response to solar irradiance reduction; it also received the highest participation of all GeoMIP experiments thus far. Most models have been modified since CMIP5, so evaluating climate response to G1 with the new model versions could serve as a useful comparison. A longer simulation will also improve the detection of changes in extreme events and modes of climate variability, particularly as related to regional changes. Moreover, some processes of interest, such as changes in ice sheet dynamics or deep ocean circulation, take longer than 50 years to resolve. Although 100 years is probably an insufficient length of time to fully assess changes in these fields, it may nevertheless allow enough time for an early indication of features that emerge above the noise level of the climate system; early detection will be aided by having multiple ensemble members.

G1ext will be highly synergistic with the Cloud Feedback Model Intercomparison Project (CFMIP). In particular, CFMIP plans to include an experiment in which total solar irradiance is abruptly increased or decreased by a constant amount that is similar to the amount of total solar irradiance decrease in G1ext. Through comparisons between these CFMIP experiments and G1ext, we will be able to better separate rapid adjustments and feedback responses to radiative forcing. These experiments will also reveal key information on the differences in cloud responses to single vs. combined forcings, which has strong implications for diagnosing transient and equilibrium climate sensitivity.

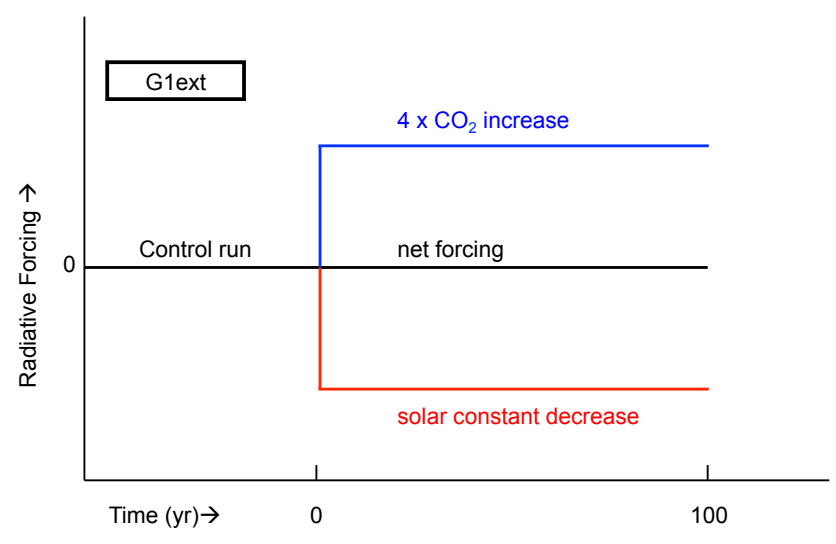

Figure 1. Schematic of experiment G1ext. The experiment is started from a preindustrial control run. The instantaneous quadrupling of the $\mathrm{CO}_{2}$ concentration from its preindustrial value is balanced by a reduction in solar irradiance for 100 years.

G1 is the only original experiment from Kravitz et al. (2011) that is proposed to be lengthened. The climate responses in the other original simulations have lower signalto-noise ratios, so extending these simulations is a lower priority at present.

\subsection{G6sulfur}

Previous GeoMIP experiments (G3 and G4) used RCP4.5 (Representative Concentration Pathway 4.5) as a background scenario. To maintain relevance to the newly designed experiments in CMIP6, our background scenario is changed to follow the ScenarioMIP Tier 1 scenarios, described above.

Under experiment G6sulfur (Fig. 2), stratospheric sulfate aerosol precursors will be injected into the model with the goal of reducing the magnitude of the net anthropogenic radiative forcing from the ScenarioMIP Tier 1 high forcing scenario to match that of the ScenarioMIP Tier 1 medium forcing scenario (decadal means should be within $\pm 0.1 \mathrm{~W} \mathrm{~m}^{-2}$ ). The motivation for this choice is to evaluate a climate in which geoengineering is used to only partially offset climate change, which would hopefully reduce the burden of adaptation. The choice of the medium forcing scenario as the target, instead of the low forcing scenario (as in Sect. 4.1), is because the required amount of sulfate aerosol injection to achieve a low anthropogenic forcing is quite large. Representing such large values of injection in a variety of climate models will likely lead to highly variable inter-model results that are overly sensitive to individual parameterizations.

For this experiment, geoengineering will be simulated over years 2020-2100. All atmospheric constituents in the ScenarioMIP Tier 1 scenarios are well defined through the year 2100. Some modeling groups may have an internal sulfate aerosol treatment; the radiative response to stratospheric sulfate aerosol injection should be determined in each model so the proper amount of aerosol is injected into the model such 


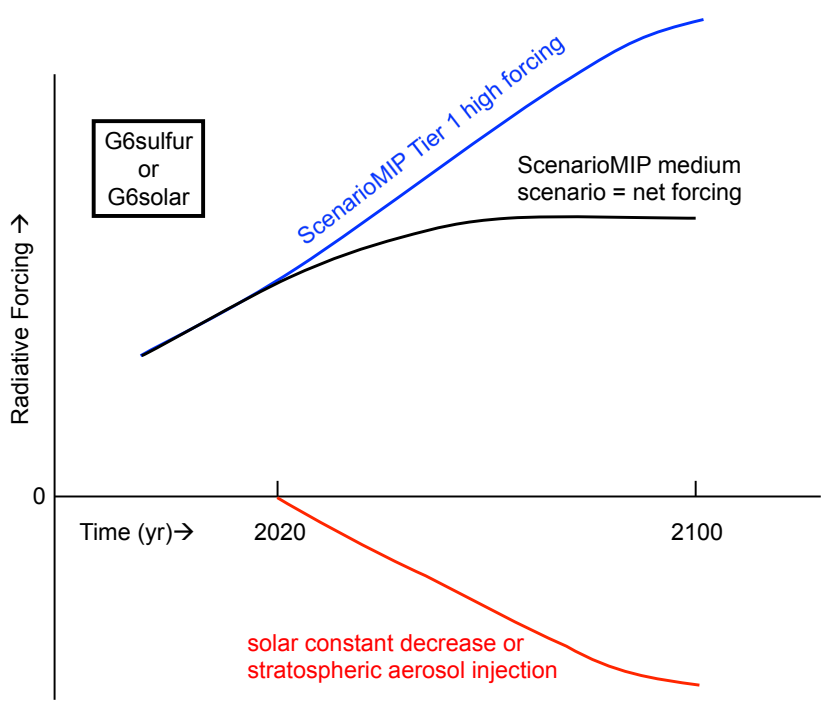

Figure 2. Schematic of experiments G6sulfur and G6solar. Against a background of the ScenarioMIP Tier 1 high forcing scenario, geoengineering will be conducted at time-varying amounts to return net anthropogenic radiative forcing to the levels of the ScenarioMIP Tier 1 medium forcing scenario. Geoengineering will be accomplished by stratospheric aerosol injection (G6sulfur) or solar irradiance reduction (G6solar).

that the total radiative forcing objectives of the experiment are met. This procedure will be more difficult for models that have a complex microphysical treatment of the aerosols, which may require more sophisticated methods of meeting the goals of G6sulfur. One method to calculate the necessary amount of sulfate aerosol is a double radiation call, once with and once without the stratospheric aerosols. Another potential method involves using feedback methods (Jarvis and Leedal, 2012; Kravitz et al., 2014b; MacMartin et al., 2014). For models that have no dynamical treatment of sulfate aerosols, GeoMIP will provide a data set of aerosol optical depth, as well as ozone fields that are consistent with this aerosol distribution; these fields will be consistent with the fields generated for G4SSA (see Sect. 3.2 for further details). The amount of sulfate injection needed for a given model to achieve the goals of this experiment may vary, so modeling groups should scale the aerosol and ozone perturbation fields as necessary.

Of notable importance is that the life cycle of stratospheric sulfate aerosols is very complex. To date, there are no comprehensive simulations of stratospheric sulfate aerosol geoengineering that include aerosol microphysical processes, explicit size representation, interactive chemistry, clouds, and radiation. Of the more comprehensive simulations conducted, some studies include aerosol microphysics and explicit size representation but do not allow oxidants to evolve (e.g., Heckendorn et al., 2009) or do not allow aerosol heating to interact with radiation and dynamics (e.g., English et al., 2012). Other studies include aerosol microphysics and heating but represent the aerosol size distribution in assumed lognormal modes of prescribed constant width (e.g., Niemeier et al., 2011, 2013). Because geoengineering has not been conducted in the real world, there are no observations to constrain these particular physical processes in models. Kokkola et al. (2009) showed that even for volcanic eruptions, capturing the evolution of the aerosol size distribution is more difficult for larger amounts of stratospheric $\mathrm{SO}_{2}$ injection. An additional complicating factor is that stratospheric aerosol geoengineering would be expected to modify the quasi-biennial oscillation (Aquila et al., 2014). This is important for the direct effects on circulation as well as the fact that the phase of the quasi-biennial oscillation would affect the rate of meridional transport of stratospheric aerosols (Plumb and Bell, 1982). Development of models that can represent these processes and thus constrain the uncertainties that may arise is ongoing, and we expect that substantial progress will be made by the time the GeoMIP6 experiments will begin. Nevertheless, the goal of GeoMIP is to use the best available models and attempt to characterize uncertainties introduced by structural uncertainties in those models.

All simulations will be conducted as if the aerosols or aerosol precursors are emitted in a line from $10^{\circ} \mathrm{S}$ to $10^{\circ} \mathrm{N}$ along a single longitude band $\left(0^{\circ}\right)$. This setup differs somewhat from a single point source injection in that it allows models with a strong stratospheric transport barrier to achieve a reasonable global distribution of sulfate aerosol rather than an aerosol optical depth maximum in the tropics. The size of the injection zone can substantially alter the resulting aerosol size distribution (English et al., 2012). Indeed, inter-model differences in the resulting spatial distributions of sulfate aerosols, and hence aerosol forcing, will be a key focus of analysis for this experiment. However, we do not wish to add additional complications to the simulation design at this time, so our design does not strongly deviate from the design of a point-source injection. Injected aerosols or aerosol precursors should be evenly spread across model layers between 18 and $20 \mathrm{~km}$. This is a slightly different setup from that of the original sulfate aerosol experiments (Kravitz et al., 2011), but sedimentation processes and self-lofting due to heating are likely to result in the aerosols being distributed between 16 and $25 \mathrm{~km}$ in altitude, which is the specification of the original experiments. Models will use their own individual treatments of aerosol optical properties, as this would be too difficult to specify in a consistent way across all participating models.

\subsection{G6solar}

Experiment G3solar was proposed as an unofficial counterpart to experiment G3 (Kravitz et al., 2011; Table 1); in G3solar, the goals of G3 were achieved using a solar irradiance reduction rather than stratospheric sulfate aerosol injections. Comparison of these two simulations would reveal differential effects of sulfate aerosols and solar irradiance 
reduction. Preliminary results from a limited set of models show some differences in the results of the two experiments, particularly related to the hydrological cycle response (Niemeier et al., 2013).

We propose G6solar as a parallel experiment to G6sulfur, to compare the effects of solar reduction with those of stratospheric aerosols. G6solar uses the same setup as G6sulfur, but geoengineering is performed using solar irradiance reduction (Fig. 2). In particular, the inter-model differences in the spatial distribution of forcing are likely to be smaller than in G6sulfur, providing a useful context on the effects of uncertainties in stratospheric sulfate aerosol transport.

\subsection{G7cirrus}

A recent proposal in the geoengineering literature is the idea of seeding cirrus clouds, thinning them and thus allowing more longwave radiation to escape to space (Mitchell et al., 2009; Storelvmo et al., 2013). Encapsulated in this idea are two complementary areas of investigation: (1) the experimental design should capture the dominant effect of a drying of the upper troposphere (Muri et al., 2014), and (2) the experiment should allow for a determination of the effects on future climate response to geoengineering via cirrus thinning.

Because different models have different treatments of cirrus clouds, the description of the experimental design (below) consists of a simple treatment of cirrus clouds, allowing all models to simulate this experiment in the same way. Therefore, this experiment can be seen as assessing the spread of model response to a simple sensitivity test that mimics a proposed geoengineering technique. As such, this concept is directly relevant to answering questions about the sensitivity of ice clouds to perturbations, which directly impacts changes in convection, circulation, and ultimately climate sensitivity. In particular, by simulating this experiment in fully coupled general circulation models, we can ascertain both how forced changes in high clouds affect circulation and the radiation budget and, in turn, how those effects feedback onto changes in high cloud coverage. In particular, different models will likely have different geographical distributions of radiative forcing, which will be a focus of future analyses. This experiment will complement results obtained through CFMIP dealing with isolating the effects of cloud-radiation interactions in ice clouds.

The goal of cirrus seeding in the real world would be to cause cirrus clouds to consist of fewer but larger ice crystals, thus increasing the fall speed and reducing the infrared opacity of these clouds. A first attempt at representing the effects of cirrus cloud thinning was to multiply cirrus cloud optical depth in the radiation code by a factor of $\varepsilon<1$ without modifying the actual cirrus fields. However, modifying cirrus optical depth in this way could be difficult in some models, as many models only distinguish between liquid and ice clouds. A specification that most models can handle would be to im-

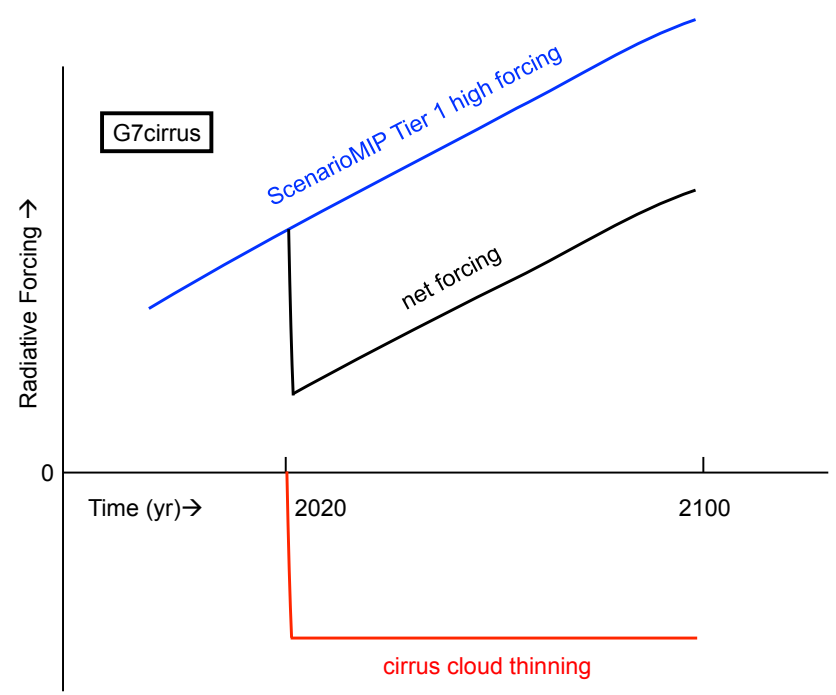

Figure 3. Test simulations of reducing cirrus cloud optical depth ( $\tau$ ) as described in Sect. 2.4. $\tau$ were scaled by a factor of $\varepsilon<1$ ( $x$ axis). The amount of surface air temperature change due to this scaling ( $y$ axis) was measured over a 4-year average; 0 indicates the global mean surface air temperature over years 2020-2023 in an RCP8.5 simulation. All simulations were performed using GISS ModelE2 (Schmidt et al., 2014).

plement the factor $\varepsilon$ in ice clouds with temperature below $-35^{\circ} \mathrm{C}$ and pressures lower than $600 \mathrm{hPa}$. This will not account for models that formulate the effects of cirrus clouds in the infrared as a modification to atmospheric emissivity, not optical depth.

Figure 3 shows results from GISS (Goddard Institute for Space Studies) ModelE2 (Schmidt et al., 2014) for various values of $\varepsilon$ when applied to ice clouds with temperature below $-35^{\circ} \mathrm{C}$ and pressures lower than $600 \mathrm{hPa}$. Global mean surface air temperature changes appear to be linear with $\varepsilon$, but the amount of cooling is quite small. We hypothesize that these results are due to cirrus clouds being very efficient absorbers of longwave radiation, even if they are optically thin. To achieve substantial cooling, it appears necessary to reduce cirrus cloud coverage, not just optical depth. Single model simulations of cirrus thinning that incorporate a treatment of cloud microphysics show more substantial surface cooling. Crook et al. (2015) and Muri et al. (2014) both found global cooling of approximately $1{ }^{\circ} \mathrm{C}$ (using a full ocean and a slab ocean, respectively), and Storelvmo et al. (2014) found global mean cooling of $1.4^{\circ} \mathrm{C}$ (using a full ocean) in simulations of high-latitude cirrus cloud thinning. As such, we conclude that the simplistic method of decreasing cirrus cloud optical depth does not capture the relevant effects necessary to represent cirrus cloud thinning.

A representation of ice microphysics appears to be important in representing cooling due to cirrus ice thinning. Storelvmo et al. $(2013,2014)$ conducted cirrus thinning experiments using the complex cirrus parameterization of Bara- 


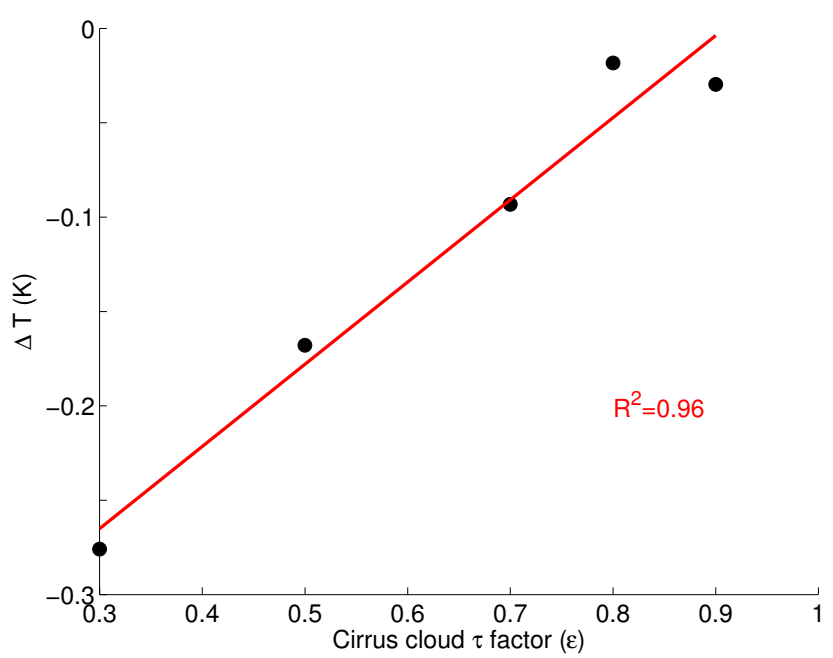

Figure 4. A sensitivity study of the effects of changing cirrus ice crystal sedimentation velocity in NorESM1-ME. vfx2, vfx4, and vfx 8 indicate an increase in the sedimentation velocity by 2,4 , and 8 times, respectively. $y$ axis shows the global mean temperature change as a function of year ( $x$ axis); differences are calculated with respect to an average over years 2050-2055 under an RCP8.5 scenario.

hona and Nenes $(2008,2009)$. Muri et al. (2014) used a simpler approach, wherein ice crystal fall speed was increased in the Community Earth System Model version 1.0.3 (Hurrell et al., 2013). They found that the prominent climate effects of cirrus thinning are well approximated by simply increasing cirrus ice sedimentation velocity. The ice crystal fall speed is known to have strong effects on climate; the ECHAM family of models use it as a model tuning parameter (Roeckner et al., 2003). Simulations using NorESM1-ME (Norwegian community Earth System Model; Tjiputra et al., 2013) also indicate a strong global mean temperature response to changes in fall speed (Fig. 4). In addition, Fig. 5 shows that for an eight-fold increase of the ice crystal fall speed against a background of RCP8.5, relative humidities in the upper troposphere are reduced by over $30 \%$ in the tropical upper troposphere, which is consistent with the aims of cirrus cloud thinning. We conclude that increasing the ice sedimentation velocity has a strong effect, can be reproduced in multiple models, and captures the concept of cirrus thinning. As such, this is the method that should be adopted by all models participating in G7cirrus: all modeling groups should add a new local variable that replaces (in all locations where temperature is colder than $235 \mathrm{~K}$ ) the ice mass mixing ratio in the calculation of the sedimentation velocity with a value that is 8 times the original ice mass mixing ratio. This methodology has an added co-benefit, in that it is a sensitivity test involving parameter perturbations; the results of this experiment could be informative for other model intercomparison projects like CFMIP.

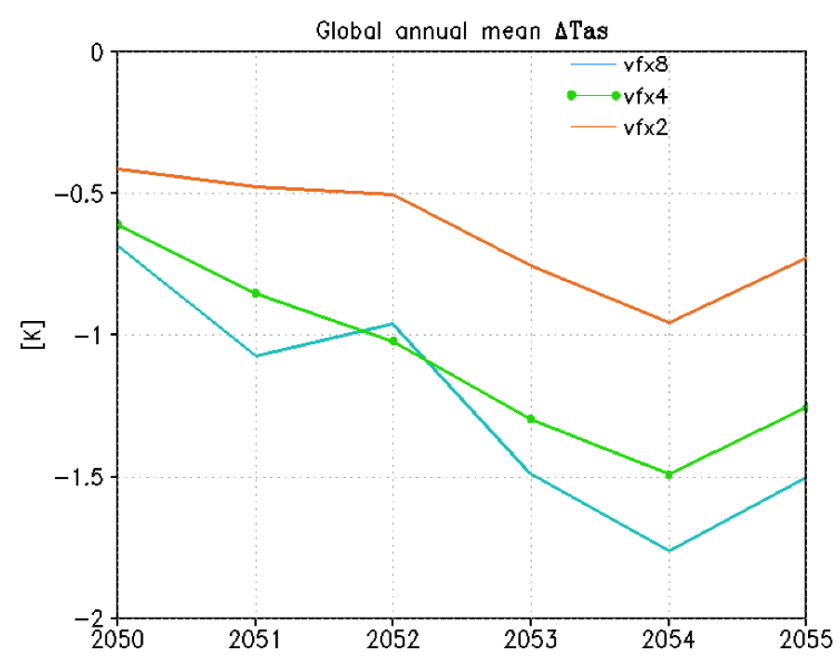

Figure 5. Zonally averaged annual mean of the difference in relative humidity (\%) from NorESM1-ME for an octupling of the cirrus ice crystal fall speed. Differences are calculated as an average over years 2050-2055 against a background of RCP8.5.

Increasing fall speed is not a perfect representation of cirrus cloud thinning, as fall speed is greater for large crystals. Actually introducing ice nuclei (IN) would result in large ice crystals (although not so large as to fall out quickly), but increasing the fall speed causes all large crystals to fall out quickly, resulting in an unrealistically small size distribution of crystals. Doubling the size of the ice crystals would be a better representation of cirrus cloud seeding, but how best to double a size distribution is not well defined. Moreover, a change in size of the ice crystals would change the scattering properties of the crystals; accounting for this effect in a way that is consistent across all participating models would be quite complicated. Liu et al. (2012) found that homogeneous ice nucleation plays an important role in cirrus cloud crystal formation; our focus on the homogeneous nucleation regime (temperatures colder than $235 \mathrm{~K}$ ) improves the confidence in our ability to represent many of the effects of cirrus thinning.

Storelvmo and Herger (2014) found that the majority of the cirrus thinning effects on net cloud forcing and surface temperatures are due to cirrus seeding outside of the tropics; including the tropics in the regions that are seeded caused a modest additional effect. However, so as not to introduce artificial boundaries in the regions where cirrus clouds are altered, cirrus clouds will be modified at all latitudes.

The design of G7cirrus (Fig. 6) is comparable to previous GeoMIP experiments. Against a background of the ScenarioMIP Tier 1 high forcing scenario, cirrus seeding will begin in 2020 and continue through the year 2100 . The goal of this experiment is to seed cirrus by a constant amount that reduces average global mean temperature in the decade 2020-2029 to that of the decade 1970-1979 (as calculated in a historical run), offsetting a radiative forcing of approx- 


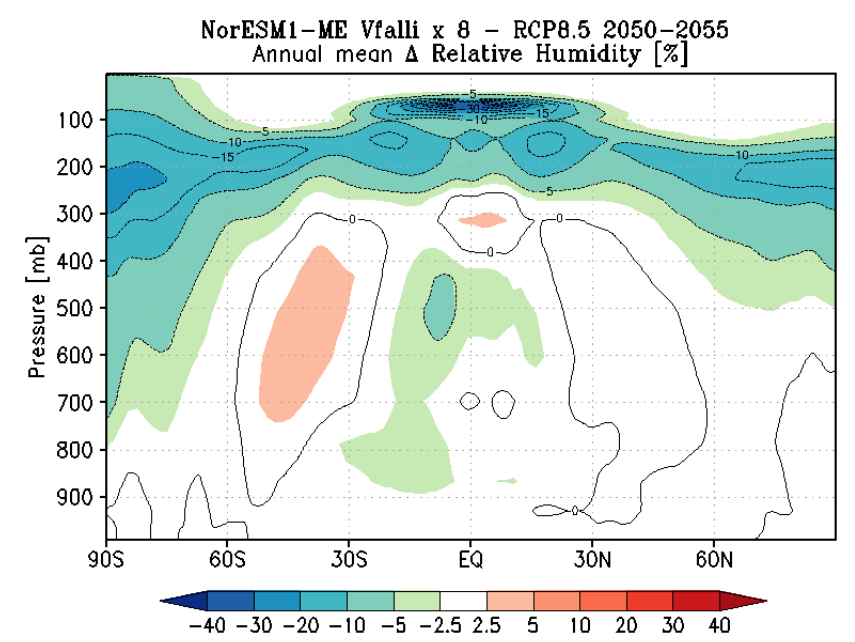

Figure 6. Schematic of experiment G7cirrus. Against a background scenario of the ScenarioMIP Tier 1 high forcing scenario, a representation of cirrus cloud seeding will reduce net forcing by a constant amount. This simulation will begin in 2020 and will be conducted for 80 years.

imately $1.0 \mathrm{~W} \mathrm{~m}^{-2}$. The decade $1970-1979$ was chosen to avoid the climate effects of the 1982 El Chichón eruption, the 1991 Mount Pinatubo eruption, and the unusually large El Niño events in 1982 and 1998. Unlike G6sulfur or G6solar, G7cirrus does not propose to return net radiative forcing from one ScenarioMIP Tier 1 scenario to another, as it is yet unclear what levels of forcing could be achieved through cirrus seeding.

Cirrus cloud processes are poorly understood and poorly represented in climate models. As an example, comparisons between observed and modeled ice water path in CMIP5 models reveal model biases of a factor of 2-10 ( $\mathrm{Li}$ et al., 2012). Nevertheless, preliminary results and recent studies indicate that G7cirrus will reveal commonalities among model responses. Therefore, in addition to providing relevant information about the potentials and limitations of cirrus thinning, exploring inter-model differences in the results can reveal sources of model biases, directly addressing one of the core scientific questions in CMIP6.

\section{Tier 2 experiments in GeoMIP6}

In addition to the four Tier 1 experiments, we propose another set of experiments that will aid in diagnosing climate model response.

\subsection{Time-slice simulations}

Separately calculating the rapid adjustments and the feedback response (also called the fast and slow responses, respectively) can reveal fundamental climate behavior. This has been shown to be particularly useful for geoengineer- ing simulations (Tilmes et al., 2013; Kravitz et al., 2013b; Huneeus et al., 2014). As such, we are requesting that all participating modeling groups conduct time-slice simulations (e.g., Cubasch et al., 1995) for each of the Tier 1 experiments to aid in diagnosing radiative forcing for the scenarios proposed here. These simulations will provide key information about the climate system response to radiative forcing, as well as the relative sensitivities of climate responses and model biases to changes in aerosol and cloud microphysical properties, thus directly addressing several of the core science questions in CMIP6.

These time-slice experiments involve fixed sea surface temperature (SST) simulations for a period of 10 years; these are similar to radiative flux perturbation simulations (Haywood et al., 2009). In these simulations, SSTs, sea ice, and all boundary conditions (greenhouse gas concentrations, aerosols, and other climate forcing agents) are to be prescribed at a constant climatology for the entire 10-year simulation. In most of the time-slice simulations, an external forcing is applied. For this forcing, the climatology is derived from the appropriate geoengineering experiment. For all the other boundary conditions, the climatologies are derived from the appropriate reference scenarios, in which no geoengineering is applied. Each Tier 1 experiment will have two associated time-slice simulations, one at the beginning of the coupled simulation and one at the end. The time-slice simulations are described in more detail in Table 2.

\subsection{G4-specified stratospheric aerosol experiment (G4SSA)}

There are several issues in simulations of geoengineering with prognostic stratospheric sulfate aerosols, as differences in the resulting aerosol distribution can have prominent effects on the climate impacts of geoengineering and thus can produce large differences in the response between the models. To remove this difference between the models, Tilmes et al. (2015) have designed an experiment for chemistry climate models (CCMs) called G4SSA. This experiment is designed so that all models can use the same prescribed stratospheric sulfur distribution, allowing for assessments of the range of climate responses for different representations of aerosol-chemistry and climate interactions. This experiment is connected to the other experiments in the CCMI.

The experiment design takes inspiration from GeoMIP experiment G4. Against a background of RCP6.0, a layer of stratospheric aerosols will be injected into the model at a rate of $8 \mathrm{TgSO}_{2}$ year $^{-1}$. Instead of allowing the models to calculate their aerosol distributions, a distribution of surface area density and other aerosol parameters will be provided to all models. The described distribution can also be scaled so as to apply to other scenarios, such as the ScenarioMIP scenarios (this is relevant for experiment G6sulfur). We will provide time series of aerosol optical depth and ozone concentration that are consistent with the aerosol 
Table 2. Time-slice simulations associated with each of the four Tier 1 experiments. Further description of the time-slice simulations is given in Sect. 3.1. Each Tier 1 has two associated time-slice simulations: one for the beginning of the coupled simulation and one at the end of the coupled simulation. The first time-slice simulations for G6sulfur and G6solar are identical, as no geoengineering has been applied yet. As such, this simulation is simply called G6Slice1.

\begin{tabular}{lll}
\hline Experiment name & Applied forcing & Boundary conditions \\
\hline G1extSlice1 & $4 \times \mathrm{CO}_{2}$ & piControl \\
G1extSlice2 & $4 \times \mathrm{CO}_{2}$ & abrupt4xCO2 after 100 years \\
G6Slice1 & None & ScenarioMIP Tier 1 high forcing scenario in year 2020 \\
G6sulfurSlice2 & G6sulfur in year 2100 & ScenarioMIP Tier 1 high forcing scenario in year 2100 \\
G6solarSlice2 & G6solar in year 2100 & ScenarioMIP Tier 1 high forcing scenario in year 2100 \\
G7cirrusSlice1 & G7cirrus in year 2020 & ScenarioMIP Tier 1 high forcing scenario in year 2020 \\
G7cirrusSlice2 & G7cirrus in year 2100 & ScenarioMIP Tier 1 high forcing scenario in year 2100 \\
\hline
\end{tabular}

distribution at the website https://www2.acd.ucar.edu/gcm/ geomip-g4-specified-stratospheric-aerosol-data-set.

Although G4SSA was developed for CCMs, it would be useful to obtain results from general circulation models (GCMs) or Earth system models (ESMs) as well, hence the inclusion in GeoMIP6. These two classes of models have very different treatments of the atmosphere, including stratospheric chemistry, aerosol microphysics, and representation of the quasi-biennial oscillation. As examples, CCMs generally have more thorough treatments than ESMs of stratospheric chemistry, transport, and aerosol microphysics, but they have less thorough treatments of cloud microphysics and atmosphere-ocean coupling. Comparisons between CCMs and ESMs can reveal whether complex treatments of some of these processes have large effects on the answers obtained. As such, these comparisons can reveal some of the mechanisms behind the climate model response to stratospheric aerosol geoengineering and provide a guideline for identifying which model representations of physical processes need improvement.

\subsection{Overshoot scenarios: G6sulfurExt and G6solarExt}

ScenarioMIP includes an overshoot scenario (Boucher et al., 2012). In this experiment, beginning from the ScenarioMIP Tier 1 highest forcing scenario, aggressive emissions reductions beginning in the year 2100 would linearly reduce net anthropogenic emissions from those of the highest forcing scenario to those of the lowest forcing scenario. Analysis of this scenario will provide information on any potential hystereses in the simulated Earth system response and could provide warnings about potential tipping points or irreversible changes. As emissions reductions occur over the 22nd and 23rd centuries, the overshoot scenario would be an extension of the Tier 1 high forcing scenario to the year 2300. It is worth noting that the decline in forcing over the 22nd and 23rd centuries will not be linear, and the forcing level would be higher than in the lowest forcing scenario. Details on the actual forcing will be provided by the coordinators of ScenarioMIP.
Here we propose extensions of G6sulfur and G6solar that parallel the ScenarioMIP overshoot scenario; these simulations are similar to those described by Wigley (2006). The general principle behind these proposed extensions is that, at any time that the net forcing is greater in magnitude than that of the ScenarioMIP Tier 1 medium forcing scenario, geoengineering is used to reduce the net forcing. This would effectively result in a situation in which the magnitude of geoengineering is ramped up at the beginning of the simulation (before 2100, when the overshoot scenario starts). It is then ramped down near the end of the simulation once emissions reductions have sufficiently reduced the forcing from the level in the high forcing scenario, such that geoengineering would no longer be required to meet the forcing objective. This scenario will illuminate the extent to which geoengineering may help in preventing irreversible changes in the climate and avoiding tipping points.

\section{The GeoMIP Testbed}

A new feature of GeoMIP is termed the GeoMIP Testbed. This is a set of experiments that are potentially useful geoengineering studies that have been proposed by individual groups. The idea is that each group understands the key problems in its own sector and is thus uniquely posed to design a simulation that would best address those problems. That simulation design would then be vetted by individual models before a decision is be made as to whether the simulation should be undertaken by the full model suite.

The following experiments have already been proposed to be included in the GeoMIP Testbed. Additional proposals should be made to the coordinators of GeoMIP via email to the corresponding author of this publication.

\subsection{G6sulfur_limits}

Experiment G6sulfur is designed to reduce radiative forcing in a high emissions scenario to that of a moderate emissions scenario via simulation of stratospheric sulfate aerosol injection. This experiment would be useful in assessing the effec- 
tiveness of geoengineering as part of a portfolio of responses to climate change. However, this experiment only addresses one potential scenario, i.e., using geoengineering to achieve the forcing from a "medium" scenario. Increasing amounts of stratospheric $\mathrm{SO}_{2}$ injection would cause particles to coagulate and fall out more rapidly. Therefore, the relationship between the amount of injection and the resulting radiative forcing is projected to be sublinear. This problem prompts a natural question: how would the injection amount and the results from that injection differ if geoengineering were used to achieve a larger radiative forcing? This question is the first step in assessing any potential practical limits to stratospheric aerosol injection.

A natural first step in addressing this problem would involve a similar setup to that of G6sulfur. Against a background of the ScenarioMIP Tier 1 high forcing scenario, sulfate aerosol precursors would be injected into the stratosphere in sufficient amounts to reduce anthropogenic radiative forcing from the levels in the high forcing scenario to levels in the low forcing scenario. As the low forcing scenario is a ScenarioMIP Tier 1 experiment, it would likely be conducted by all GeoMIP participants, and the extra simulations would be done with relatively little preparation.

Figure 7 shows the required amount of stratospheric aerosol injection to achieve given amounts of radiative forcing; these simulations were performed in MPI-ESM-LR (Max Planck Institute ESM Low Resolution), a fully coupled general circulation model of Earth's climate. Stratospheric aerosol optical depths were prescribed from simulations conducted with ECHAM-HAM (Stier et al., 2005; Niemeier et al., 2011), a general circulation model coupled to an aerosol microphysical model that simulates the physical evolution and particle growth of sulfate aerosols. Niemeier and Timmreck (2015), who simulated both aerosol optical depth and radiative forcing in ECHAM-HAM, found that radiative forcing was approximately $10 \%$ smaller in magnitude than is reported in Fig. 7, giving an indication of the importance of circulation and stratospheric transport.

The sublinear relationship between injection amount and radiative forcing is clearly illustrated; this qualitative feature is seen in both Fig. 7 and the internally consistent treatment of Niemeier and Timmreck (2015). According to the results in Fig. 7, the difference between RCP8.5 and RCP2.6 in the year 2100 is $5.9 \mathrm{~W} \mathrm{~m}^{-2}$, or the approximate radiative forcing of a tripling of the preindustrial $\mathrm{CO}_{2}$ concentration; this difference is similar to the expected difference in forcing between the ScenarioMIP Tier 1 high forcing scenario and the Tier 1 low forcing scenario, when those scenarios are finalized. Extrapolating from the results of Fig. 7, achieving this radiative forcing would require an injection of 40$50 \mathrm{Tg} \mathrm{S}_{\text {year }}{ }^{-1}\left(80-100 \mathrm{Tg} \mathrm{SO}_{2}\right)$. This injection rate is equivalent to four to five 1991 Mount Pinatubo eruptions per year. The purpose here is to gain a multi-model perspective for multiple points on the curve in Fig. 7, thereby understand-

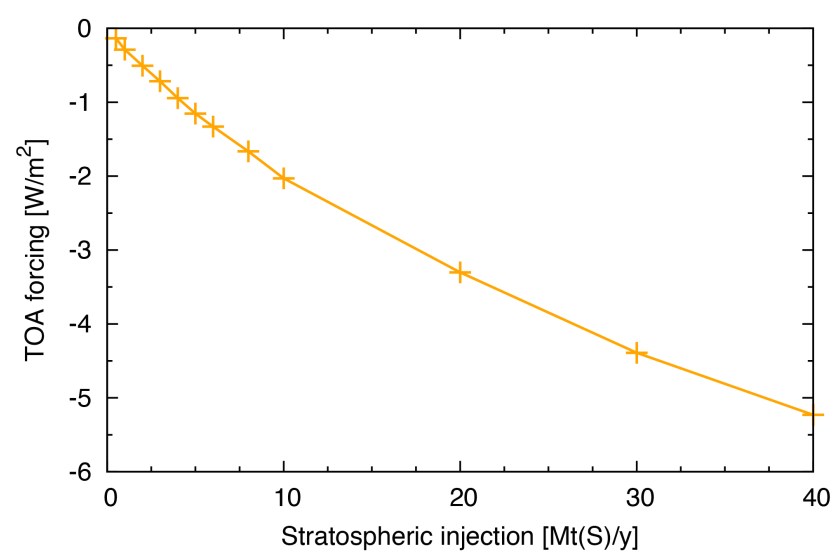

Figure 7. This figure shows the amount of annual stratospheric injection ( $x$ axis) required to offset a given level of TOA net radiative flux imbalance ( $y$ axis) in MPI-ESM-LR, a general circulation model of Earth's climate. Sulfate aerosol optical depth distributions were prescribed in the model from ECHAM5-HAM, an atmospheric general circulation model with a treatment of the microphysical evolution of sulfate aerosols. Maintaining 2020 values of net TOA radiative flux imbalance against a background of RCP8.5 requires an injection of approximately $70 \mathrm{Tg} \mathrm{S}$ year $^{-1}$ in 2100 (based on extrapolation of the above values). All values were calculated for injection of $\mathrm{SO}_{2}$ into one grid box over the Equator; other injection strategies would likely require a different injection rate to achieve the same radiative forcing. Results differ somewhat from those of Niemeier and Timmreck (2015), who describe radiative forcing results solely from ECHAM5-HAM; their treatment of the aerosols is fully internally consistent.

ing the range of required injection amounts to achieve this experiment's goal.

\subsection{GeoSulfur10, GeoSulfur20, GeoSulfur50}

A different way of quantifying the effects of stratospheric aerosol geoengineering is to perform a series of experiments in which the hypothetical rate of injection of stratospheric sulfate aerosols is constrained. Such a simulation would be well suited to ascertain the range of model responses to a fixed amount of $\mathrm{SO}_{2}$ injection, highlighting model diversity. Against a background of the ScenarioMIP Tier 1 high forcing scenario, the modeling groups will inject 10,20 , or $50 \mathrm{Tg} \mathrm{SO}_{2}$ year $^{-1}$ into the lower stratosphere, in a similar setup to experiment G4 (Kravitz et al., 2011).

\subsection{GeoLandAlbedo}

Experiment G1ocean-albedo has simulated the effects of marine cloud brightening by increasing ocean albedo by a constant multiplication factor (Kravitz et al., 2013a). However, GeoMIP has not yet explored land-based approaches towards solar radiation management. Such approaches could readily be implemented on the regional scale, as human activities already control the albedo of a significant fraction of the land 
surface. We therefore propose an alternative experiment in which the land surface albedo is increased, against a background of the CMIP5 abrupt4xCO2 experiment.

Under experiment GeoLandAlbedo, the land surface albedo would be increased by a uniform amount of 0.1 across all urban and agricultural areas. Such an increment represents a reasonable estimate of the maximum large-scale albedo increase that could be achieved in practice (Lobell et al., 2006; Lenton and Vaughan, 2009; Davin et al., 2014). The aim of experiment GeoLandAlbedo would not be to achieve a global energy balance but rather to determine the extent to which land surface albedo changes could offset the effects of increasing greenhouse gases on a regional basis.

To some degree, different aspects of this problem have been explored. Irvine et al. (2011) determined that different types of surface albedo geoengineering were incapable of offsetting the radiative forcing from a doubling of the $\mathrm{CO}_{2}$ concentration, and the adverse side effects of such attempts could be large. Focusing only on bio-engineering crops to increase crop canopy albedo (Ridgwell et al., 2009) could cause local cooling effects (Doughty et al., 2011) but would likely have a small global impact (Singarayer et al., 2009; Singarayer and Davies-Barnard, 2012).

All of the previous studies on terrestrial-based albedo increases were conducted with single models, so the robustness of the effectiveness of this particular method of geoengineering, as well as the side effects, have not yet been tested. Assessing the range of responses to terrestrial-based geoengineering is especially important, given the wide range of structural and parametric uncertainties associated with modeling land surface processes.

\section{Conclusions}

The climate model experiment designs presented here mark the beginning of a concerted effort to include broader perspectives within GeoMIP. The extension of all experiments to at least 80 years is recommended to obtain more robust estimates of changes in extremes and modes of variability; it will be particularly interesting to discover what results can be obtained from G1ext that could not be obtained through analyses of experiment G1, particularly in relation to extreme events (Curry et al., 2014) and modes of climate variability (Gabriel and Robock, 2015). The two G6 experiments were designed to open the door toward possible conversations with designers of climate change scenarios. We have begun to explore potential synergies with ScenarioMIP, on which our core simulations are based. In addition, by standardizing designs, we have provided future avenues for a G6like experiment looking at sea spray geoengineering, an experiment we have chosen not to include at present, as the previous sea spray geoengineering experiments (Kravitz et al., 2013a) are still being analyzed. Experiment G7cirrus is the first model intercomparison of the new idea of cirrus thinning and is designed to open avenues of investigation in both geoengineering and cirrus cloud microphysical representations. G4SSA was designed to explore commonalities and differences between general circulation models and CCMs, potentially highlighting processes that are important in representing the effects of aerosols not only on atmospheric chemistry, but also on dynamics and climate.

Geoengineering has the potential to impact climate systems at all scales, so, by incorporating requirements from communities studying these different systems, we can broaden the usefulness of GeoMIP to a wider variety of scientists, policymakers, and other stakeholders. The GeoMIP Testbed is a key part of this effort. Under this new framework, individual communities can propose and test experiments that are designed to address problems in their sectors, providing invaluable information as to whether simulations by the full GeoMIP community are warranted.

Nevertheless, there remain some key gaps in GeoMIP that can provide a roadmap for future experiment design. One notable area is in impacts assessment. GeoMIP is quite adept at calculating expected climate effects from particular geoengineering scenarios, but translating those effects into impacts on people has only been explored in a limited set of studies (e.g., Xia et al., 2014). Interaction with the impacts assessment communities is one of the highest priorities for future directions of GeoMIP. This is particularly applicable for effects on developing countries, many of which will be most affected by climate change and thus might also be most affected by geoengineering.

Another notable gap is the effect of geoengineering on carbon cycle feedbacks. Studies with intermediate complexity ESMs suggest that geoengineering could have a profound effect on the global carbon cycle through, for example, an enhancement of the land carbon sink (Keller et al., 2014). While much can be learned about the response of the carbon cycle to geoengineering from the experiments proposed in this article, the atmospheric carbon concentration does not evolve freely in all experiments. Multi-model studies driven by emissions which allow the atmospheric $\mathrm{CO}_{2}$ concentration to evolve freely would provide valuable insights into the effect of SRM on this important feedback (e.g., the Coupled Carbon Cycle Climate Model Intercomparison Project, or C4MIP; Friedlingstein et al., 2006).

Although we expect that this new suite of climate model experiments will be useful in addressing many uncertainties in the physical science of geoengineering, there will remain many key questions. These experiment designs are idealized and are not representative of how geoengineering might be done in the real world, if society were to decide to deploy it. These designs also do not include studies of feasibility; some of the designed strategies might be more easily implemented in the real world than others. Moreover, while physical science studies are necessary for gaining information about the effects and impacts of geoengineering, they are only one aspect among a multitude of concerns, relating to both natural 
and social sciences, that are crucial for making informed decisions about geoengineering (e.g., Robock, 2014).

Acknowledgements. We thank Thorsten Mauritsen and Erich Roeckner for input on the protocol for G7cirrus. We also thank Bjorn Stevens and the CMIP6 organizing committee for more general comments on the experimental protocol and two anonymous reviewers for their insightful comments. Ben Kravitz and Hailong Wang are supported by the Fund for Innovative Climate and Energy Research (FICER). The Pacific Northwest National Laboratory is operated for the US Department of Energy by Battelle Memorial Institute under contract DE-AC05-76RL01830. Simulations performed by Ben Kravitz were supported by the NASA High-End Computing (HEC) Program through the NASA Center for Climate Simulation (NCCS) at Goddard Space Flight Center. Alan Robock is supported by NSF grants AGS-1157525 and GEO-1240507. The National Center for Atmospheric Research is funded by the National Science Foundation. Andy Jones was supported by the Joint UK DECC/Defra Met Office Hadley Centre Climate Programme (GA01101). Helene Muri is supported by the Norwegian Research Council project EXPECT (grant no. 229760/E10) and computing time was provided by NOTUR. $\mathrm{U}$. Niemeier is supported by the priority program 1689 of the German Research Foundation within project CEIBRAL. Jana Sillmann is supported by the Norwegian Research Council project NAPEX (229778). Shingo Watanabe is supported by the SOUSEI program, MEXT, Japan.

Edited by: D. Roche

\section{References}

Alterskjær, K., Kristjánsson, J. E., Boucher, O., Muri, H., Niemeier, U., Schmidt, H., Schulz, M., and Timmreck, C.: Seasalt injections into the low-latitude marine boundary layer: The transient response in three Earth system models, J. Geophys. Res., 118, 12195-12206, doi:10.1002/2013JD020432, 2013.

Aquila, V., Garfinkel, C. I., Newman, P. A., Oman, L. D., and Waugh, D. W.: Modifications of the quasi-biennial oscillation by a geoengineering perturbation of the stratospheric aerosol layer, Geophys. Res. Lett., 41, 1738-1744, doi:10.1002/2013GL058818, 2014.

Barahona, D. and Nenes, A.: Parameterization of cirrus cloud formation in large-scale models: Homogeneous nucleation, J. Geophys. Res., 113, D11211, doi:10.1029/2007JD009355, 2008.

Barahona, D. and Nenes, A.: Parameterizing the competition between homogeneous and heterogeneous freezing in cirrus cloud formation - monodisperse ice nuclei, Atmos. Chem. Phys., 9, 369-381, doi:10.5194/acp-9-369-2009, 2009.

Berdahl, M., Robock, A., Ji, D., Moore, J. C., Jones, A., Kravitz, B., and Watanabe, S.: Arctic cryosphere response in the Geoengineering Model Intercomparison Project G3 and G4 scenarios, J. Geophys. Res., 119, 1308-1321, doi:10.1002/2013JD020627, 2014.

Boucher, O., Halloran, P. R., Burke, E. J., Doutriaux-Boucher, M., Jones, C. D., Lowe, J., Ringer, M. A., Robertson, E., and Wu, P.: Reversibility in an Earth System model in response to $\mathrm{CO}_{2}$ concentration changes, Environ. Res. Lett., 7, 024013, doi:10.1088/1748-9326/7/2/024013, 2012.

Boucher, O., Randall, D., Artaxo, P., Bretherton, C., Feingold, G., Forster, P., Kerminen, V.-M., Kondo, Y., Liao, H., Lohmann, U., Rasch, P., Satheesh, S. K., Sherwood, S., Stevens, B., and Zhang, X. Y.: Clouds and aerosols, in: Climate Change 2013: The Physical Science Basis. Contribution of Working Group I to the Fifth Assessment Report of the Intergovernmental Panel on Climate Change, edited by: Stocker, T. F., Qin, D., Plattner, G.-K., Tignor, M., Allen, S. K., Boschung, J., Nauels, A., Xia, Y., Bex, V., and Midgley, P. M., Cambridge University Press, Cambridge, UK and New York, NY, USA, 571-658, doi:10.1017/CBO9781107415324.016, 2013.

Crook, J., Jackson, L. S., Osprey, S. M., and Forster, P. M.: A comparison of temperature and precipitation responses to different Earth radiation management geoengineering schemes, J. Geophys. Res., 120, doi:10.1002/2015JD023269, 2015.

Cubasch, U., Waszkewitz, J., Hegerl, G., and Perlwitz, J.: Regional climate changes as simulated in time-slice experiments, Climatic Change, 31, 273-304, doi:10.1007/BF01095150, 1995.

Curry, C. L., Sillmann, J., Bronaugh, D., Alterskjær, K., Cole, J. N. S., Kravitz, B., Kristjánsson, J. E., Muri, H., Niemeier, U., Robock, A., and Tilmes, S.: A multi-model examination of climate extremes in an idealized geoengineering experiment, J. Geophys. Res., 119, 5226-5239, doi:10.1002/2013JD020648, 2014.

Davin, E. L., Seneviratne, S. I., Ciais, P., Olioso, A., and Wang, T.: Preferential cooling of hot extremes from cropland albedo management, P. Natl. Acad. Sci., 111, 9757-9761, doi:10.1073/pnas.1317323111, 2014.

Doughty, C. E., Field, C. B., and McMillan, A. M. S.: Can crop albedo be increased through the modification of leaf trichomes, and could this cool regional climate?, Climatic Change, 104, 379-387, 2011.

English, J. M., Toon, O. B., and Mills, M. J.: Microphysical simulations of sulfur burdens from stratospheric sulfur geoengineering, Atmos. Chem. Phys., 12, 4775-4793, doi:10.5194/acp-12-47752012, 2012.

Friedlingstein, P., Cox, P., Betts, R., Bopp, L., von Bloh, W., Brovkin, V., Cadule, P., Doney, S., Eby, M., Fung, I., Bala, G., John, J., Jones, C., Joos, F., Kato, T., Kawamiya, M., Knorr, W., Lindsay, K., Matthews, H. D., Raddatz, T., Rayner, P., Reick, C., Roeckner, E., Schnitzler, K.-G., Schnur, R., Strassmann, K., Weaver, A. J., Yoshikawa, C., and Zeng, N.: Climate-carbon cycle feedback analysis: results from the C4MIP Model Intercomparison, J. Climate, 19, 3337-3353, doi:10.1175/JCLI3800.1, 2006.

Gabriel, C. J. and Robock, A.: Stratospheric geoengineering impacts on El Niño/Southern Oscillation, Atmos. Chem. Phys. Discuss., 15, 9173-9202, doi:10.5194/acpd-15-9173-2015, 2015.

Haywood, J., Donner, L. J., Jones, A., and Golaz, J.-C.: Global indirect radiative forcing caused by aerosols: IPCC 2007: and beyond, in: Clouds in the Perturbed Climate System, edited by: Heintzenberg, J. and Charlson, R. J., MIT Press, Cambridge, 451-467, 2009.

Heckendorn, P., Weisenstein, D., Fueglistaler, S., Luo, B. P., Rozanov, E., Schraner, M., Thomason, L. W., and Peter, T.: The impact of geoengineering aerosols on stratospheric temperature 
and ozone, Environ. Res. Lett., 4, 045108, doi:10.1088/17489326/4/4/045108, 2009.

Huneeus, N., Boucher, O., Alterskjær, K., Cole, J. N. S., Curry, C. L., Ji, D., Jones, A., Kravitz, B., Kristjánsson, J. E., Moore, J. C., Muri, H., Niemeier, U., Rasch, P. J., Robock, A., Singh, B., Schmidt, H., Schulz, M., Tilmes, S., Watanabe, S., and Yoon, J.-H.: Forcings and feedbacks in the GeoMIP ensemble for a reduction in solar irradiance and increase in $\mathrm{CO}_{2}$, J. Geophys. Res., 119, 5226-5239, doi:10.1002/2013JD021110, 2014.

Hurrell, J. W., Holland, M. M., Gent, P. R., Ghan, S., Kay, J. E., Kushner, P. J., Lamarque, J.-F., Large, W. G., Lawrence, D., Lindsay, K., Lipscomb, W. H., Long, M. C., Mahowald, N., Marsh, D. R., Neale, R. B., Rasch, P., Vavrus, S., Vertenstein, M., Bader, D., Collins, W. D., Hack, J. J., Kiehl, J., and Marshall, S.: The Community Earth System Model: A framework for collaborative research, B. Am. Meteorol. Soc., 94, 1339-1360, doi:10.1175/BAMS-D-12-00121.1, 2013.

Irvine, P. J., Ridgwell, A., and Lunt, D. J.: Climatic effects of surface albedo geoengineering, J. Geophys. Res., 116, D24112, doi:10.1029/2011JD016281, 2011.

Jarvis, A. and Leedal, D.: The Geoengineering Model Intercomparison Project (GeoMIP): a control perspective, Atmos. Sci. Lett., 13, 157-163, doi:10.1002/asl.387, 2012.

Jones, A., Haywood, J. M., Alterskjær, K., Boucher, O., Cole, J. N. S., Curry, C. L., Irvine, P. J., Ji, D., Kravitz, B., Kristjánsson, J. E., Moore, J. C., Niemeier, U., Robock, A., Schmidt, H., Singh, B., Tilmes, S., Watanabe, S., and Yoon, J.-H.: The impact of abrupt suspension of solar radiation management (termination effect) in experiment G2 of the Geoengineering Model Intercomparison Project (GeoMIP), J. Geophys. Res., 118, 9743-9752, doi:10.1002/jgrd.50762, 2013.

Keller, D. P., Feng, E. Y., and Oschlies, A.: Potential climate engineering effectiveness and side effects during a high carbon dioxide-emission scenario, Nat. Commun., 5, 3304, doi:10.1038/ncomms4304, 2014.

Kleidon, A., Kravitz, B., and Renner, M.: The hydrological sensitivity to global warming and solar geoengineering derived from thermodynamic constraints, Geophys. Res. Lett., 42, 138-144, doi:10.1002/2014GL062589, 2015.

Kokkola, H., Hommel, R., Kazil, J., Niemeier, U., Partanen, A.-I., Feichter, J., and Timmreck, C.: Aerosol microphysics modules in the framework of the ECHAM5 climate model - intercomparison under stratospheric conditions, Geosci. Model Dev., 2, 97-112, doi:10.5194/gmd-2-97-2009, 2009.

Kravitz, B., Robock, A., Boucher, O., Schmidt, H., Taylor, K. E., Stenchikov, G., and Schulz, M.: The Geoengineering Model Intercomparison Project (GeoMIP), Atmos. Sci. Lett., 12, 162167, doi:10.1002/as1.316, 2011.

Kravitz, B., Forster, P. M., Jones, A., Robock, A., Alterskjær, K., Boucher, O., Jenkins, A. K. L., Korhonen, H., Kristjánsson, J. E., Muri, H., Niemeier, U., Partanen, A.-I., Rasch, P. J., Wang, H., and Watanabe, S.: Sea spray geoengineering experiments in the Geoengineering Model Intercomparison Project (GeoMIP): experimental design and preliminary results, J. Geophys. Res., 118, 11175-11186, doi:10.1002/jgrd.50856, 2013 a.

Kravitz, B., Rasch, P. J., Forster, P. M., Andrews, T., Cole, J. N. S., Irvine, P. J., Ji, D., Kristjánsson, J. E., Moore, J. C., Muri, H., Niemeier, U., Robock, A., Singh, B., Tilmes, S., Watanabe, S., and Yoon, J.-H.: An energetic perspective on hydrological cycle changes in the Geoengineering Model Intercomparison Project, J. Geophys. Res., 118, 13087-13102, doi:10.1002/2013JD020502, 2013b.

Kravitz, B., Caldeira, K., Boucher, O., Robock, A., Rasch, P. J., Alterskjær, K., Karam, D. B., Cole, J. N. S., Curry, C. L., Haywood, J. M., Irvine, P. J., Ji, D., Jones, A., Kristjánsson, J. E., Lunt, D. J., Moore, J., Niemeier, U., Schmidt, H., Schulz, M., Singh, B., Tilmes, S., Watanabe, S., Yang, S., and Yoon, J.-H.: Climate model response from the Geoengineering Model Intercomparison Project (GeoMIP), J. Geophys. Res., 118, 8320 8332, doi:10.1002/jgrd.50646, 2013c.

Kravitz, B., Robock, A., and Boucher, O.: Future directions in simulating solar geoengineering, EOS T. Am. Geophys. Un., 95, p. 280, doi:10.1002/2014EO310010, 2014a.

Kravitz, B., MacMartin, D. G., Leedal, D. T., Rasch, P. J., and Jarvis, A. J.: Explicit feedback and the management of uncertainty in meeting climate objectives with solar geoengineering, Environ. Res. Lett., 9, 044006, doi:10.1088/17489326/9/4/044006, 2014b.

Lenton, T. M. and Vaughan, N. E.: The radiative forcing potential of different climate geoengineering options, Atmos. Chem. Phys., 9, 5539-5561, doi:10.5194/acp-9-5539-2009, 2009.

Li, J.-L., Waliser, D. E., Chen, W.-T., Guan, B., Kubar, T., Stephens, G., Ma, H.-Y., Deng, M., Donner, L., Seman, C., and Horowitz, L.: An observationally based evaluation of cloud ice water in CMIP3 and CMIP5 GCMs and contemporary reanalyses using contemporary satellite data, J. Geophys. Res., 117, doi:10.1029/2012JD017640, 2012.

Liu, X., Shi, X., Zhang, K., Jensen, E. J., Gettelman, A., Barahona, D., Nenes, A., and Lawson, P.: Sensitivity studies of dust ice nuclei effect on cirrus clouds with the Community Atmosphere Model CAM5, Atmos. Chem. Phys., 12, 12061-12079, doi:10.5194/acp-12-12061-2012, 2012.

Lobell, D. B., Bala, G., and Duffy, P. B.: Biogeophysical impacts of cropland management changes on climate, Geophys. Res. Lett. 33, L06708, doi:10.1029/2005GL025492, 2006.

MacMartin, D. G., Kravitz, B., Keith, D. W., and Jarvis, A.: Dynamics of the coupled human-climate system resulting from closedloop control of solar geoengineering, Clim. Dynam., 43, 243258, doi:10.1007/s00382-013-1822-9, 2014.

MacMartin, D. G., Kravitz, B., and Rasch, P. J.: On solar geoengineering and climate uncertainty, Geophys. Res. Lett., 42, 71567161, doi:10.1002/2015GL065391, 2015.

Meehl, G. A., Moss, R., Taylor, K. E., Eyring, V., Stouffer, R. J., Bony, S., and Stevens, B.: Climate Model Intercomparisons: preparing for the next phase, EOS T. Am. Geophys. Un., 95, 7778, doi:10.1002/2014EO090001, 2014.

Mitchell, D. L. and Finnegan, W.: Modification of cirrus clouds to reduce global warming, Environ. Res. Lett., 4, 045102, doi:10.1088/1748-9326/4/4/045102, 2009.

Muri, H., Kristjánsson, J. E., Storelvmo, T., and Pfeffer, M. A.: The climatic effects of modifying cirrus clouds in a climate engineering framework, J. Geophys. Res., 119, 4174-4191, doi:10.1002/2013JD021063, 2014.

NAS: Climate Intervention: reflecting Sunlight to Cool Earth; Committee on Geoengineering Climate: technical Evaluation and Discussion of Impacts; Board on Atmospheric Sciences and Climate; Ocean Studies Board; Division on Earth and Life Studies; National Research 
Council, available at: http://www.nap.edu/catalog/18988/ climate-intervention-reflecting-sunlight-to-cool-earth (last access: 7 May 2015), 2015.

Niemeier, U. and Timmreck, C.: What is the limit of climate engineering by stratospheric injection of $\mathrm{SO}_{2}$ ?, Atmos. Chem. Phys., 15, 9129-9141, doi:10.5194/acp-15-9129-2015, 2015.

Niemeier, U., Schmidt, H., and Timmreck, C.: The dependency of geoengineered sulfate aerosol on the emission strategy, Atmos. Sci. Lett., 12, 189-194, doi:10.1002/asl.304, 2011.

Niemeier, U., Schmidt, H., Alterskjær, K., and Kristjánsson, J. E.: Solar irradiance reduction via climate engineeringimpact of different techniques on the energy balance and the hydrological cycle, J. Geophys. Res., 118, 11905-11917, doi:10.1002/2013JD020445, 2013.

O’Neill, B. C., Kriegler, E., Riahi, K., Ebi, K. L., Hallegatte, S., Carter, T. R., Mathur, R., and van Vuuren, D. P.: A new scenario framework for climate change research: the concept of shared socioeconomic pathways, Climatic Change, 122, 387400, doi:10.1007/s10584-013-0905-2, 2014.

Plumb, R. A. and Bell, R. C.: A model of quasibiennial oscillation on an equatorial beta plane, Q. J. Roy. Meteor. Soc., 108, 335352, 1982.

Ridgwell, A., Singarayer, J. S., Hetherington, A. M., and Valdes, P. J.: Tackling regional climate change by leaf albedo bio-geoengineering, Curr. Biol., 19, 146-150, doi:10.1016/j.cub.2008.12.025, 2009.

Robock, A.: Stratospheric aerosol geoengineering, Environ. Sci. Technol., 38, 162-185, 2014.

Roeckner, E., Bäuml, G., Bonaventura, L., Brokopf, R., Esch, M., Giorgetta, M., Hagemann, S., Kornlblueh, L., Schlese, U., Schulzweida, U., Kirchner, I., Manzini, E., Rhodin, A., and Tompkins, A.: The Atmospheric General Circulation Model ECHAM5, Part I: Model description, Tech. Rep. 349, available at: http://www.mpimet.mpg.de/fileadmin/models/ echam/mpi_report_349.pdf, (last access: 7 May 2015), 2003.

Schmidt, G. A., Kelley, M., Nazarenko, L., Ruedy, R., Russell, G. L., Aleinov, I., Bauer, M., Bauer, S. E., Bhat, M. K., Bleck, R., Canuto, V., Chen, Y.-H., Cheng, Y., Clune, T. L., Del Genio, A., de Fainchtein, R., Faluvegi, G., Hansen, J. E., Healy, R. J., Kiang, N. Y., Koch, D., Lacis, A. A., LeGrande, A. N., Lerner, Lo, K. K., Matthews, E. E., Menon, S., Miller, R. L., Oinas, V., Oloso, A. O., Perlwitz, J. P., Puma, M. J., Putman, W. M., Rind, D., Romanou, A., Sato, M., Shindell, D. T., Sun, S., Syed, R. A., Tausnev, N., Tsigaridis, K., Unger, N., Voulgarakis, A., Yao, M.-S., and Zhang, J.: Configuration and assessment of the GISS ModelE2 contributions to the CMIP5 archive, J. Adv. Modell. Earth Syst., 6, 141-184, doi:10.1002/2013MS000265, 2014.

Singarayer, J. S. and Davies-Barnard, T.: Regional climate change mitigation with crops: context and assessment, Philos. T. R. Soc. A, 370, 4301-4316, doi:10.1098/rsta.2012.0010, 2012.

Singarayer, J. S., Ridgwell, A., and Irvine, P.: Assessing the benefits of crop albedo bio-geoengineering, Environ. Res. Lett., 4, 045110, doi:10.1088/1748-9326/4/4/045110, 2009.
Stier, P., Feichter, J., Kinne, S., Kloster, S., Vignati, E., Wilson, J., Ganzeveld, L., Tegen, I., Werner, M., Balkanski, Y., Schulz, M., Boucher, O., Minikin, A., and Petzold, A.: The aerosol-climate model ECHAM5-HAM, Atmos. Chem. Phys., 5, 1125-1156, doi:10.5194/acp-5-1125-2005, 2005.

Storelvmo, T. and Herger, N.: Cirrus cloud susceptibility to the injection of ice nuclei in the upper troposphere, J. Geophys. Res.Atmos., 119, 2375-2389, doi:10.1002/2013JD020816, 2014.

Storelvmo, T., Kristjansson, J. E., Muri, H., Pfeffer, M., Barahona, D., and Nenes, A.: Cirrus cloud seeding has potential to cool climate, Geophys. Res. Lett., 40, 178-182, doi:10.1029/2012GL054201, 2013.

Storelvmo, T., Boos, W. R., and Herger, N.: Cirrus cloud seeding: a climate engineering mechanism with reduced side effects?, Philos. T. R. Soc. A, 372, 20140116, doi:10.1098/rsta.2014.0116, 2014.

Tilmes, S., Fasullo, J., Lamarque, J.-F., Marsch, D. R., Mills, M., Alterskjær, K., Boucher, O., Cole, J. N. S., Curry, C. L., Haywood, J. M., Irvine, P. J., Ji, D., Jones, A., Karam, D. B., Kravitz, B., Kristjánsson, J. E., Moore, J. C., Muri, H. O., Niemeier, U., Rasch, P. J., Robock, A., Schmidt, H., Schulz, M., Singh, B., Watanabe, S., Yang, S., and Yoon, J.-H.: The hydrological impact of geoengineering in the Geoengineering Model Intercomparison Project (GeoMIP), J. Geophys. Res., 118, 11036-11058, doi:10.1002/jgrd.50868, 2013.

Tilmes, S., Mills, M. J., Niemeier, U., Schmidt, H., Robock, A., Kravitz, B., Lamarque, J.-F., Pitari, G., and English, J. M.: A new Geoengineering Model Intercomparison Project (GeoMIP) experiment designed for climate and chemistry models, Geosci. Model Dev., 8, 43-49, doi:10.5194/gmd-8-43-2015, 2015.

Tjiputra, J. F., Roelandt, C., Bentsen, M., Lawrence, D. M., Lorentzen, T., Schwinger, J., Seland, Ø., and Heinze, C.: Evaluation of the carbon cycle components in the Norwegian Earth System Model (NorESM), Geosci. Model Dev., 6, 301-325, doi:10.5194/gmd-6-301-2013, 2013.

Wigley, T. M. L.: A combined mitigation/geoengineering approach to climate stabilization, Science, 314, 452-454, doi:10.1126/science.1131728, 2006.

Xia, L., Robock, A., Cole, J. N. S., Curry, C. L., Ji, D., Jones, A., Kravitz, B., Moore, J. C., Muri, H., Niemeier, U., Singh, B., Tilmes, S., Watanabe, S., and Yoon, J.-H.: Solar radiation management impacts on agriculture in China: a case study in the Geoengineering Model Intercomparison Project (GeoMIP), J. Geophys. Res., 119, 8695-8711, doi:10.1002/2013JD020630, 2014.

Yu, X., Moore, J. C., Cui, X., Rinke, A., Ji, D., Kravitz, B., and Yoon, J.-H.: Impacts, effectiveness and regional inequalities of the GeoMIP G1 to G4 solar radiation management scenarios, Global Planet. Change, 129, 10-22, doi:10.1016/j.gloplacha.2015.02.010, 2015. 